\title{
Procesos de Oxidación Avanzada y Electroquímicos para Remover Edulcorantes Artificiales del Agua
}

\author{
Advanced Oxidation and Electrochemical Processes for the \\ Removal of Artificial Sweeteners from Water
}

H. Rubí-Juárez ${ }^{1}$

${ }^{1}$ Universidad Autónoma de Ciudad Juárez

\section{RESUMEN}

El objetivo de la presente revisión es presentar investigaciones recientes sobre la remoción de algunos edulcorantes - reconocidos como contaminantes emergentes- del agua o matrices acuosas, utilizando los Procesos de Oxidación Avanzada (POA). En un intento por evadir el consumo de endulzantes calóricos que han provocado problemas de salud, los seres humanos consumen edulcorantes artificiales que prácticamente no se metabolizan después de ingerirse, de esa forma se integran al agua residual e inclusive resisten la degradación total en las plantas de tratamiento de agua convencionales. Por tanto, en bases de datos se revisaron electrooxidación, electro-Fenton, foto-Fenton, fotocatálisis heterogénea, oxidación por ultravioleta/peróxido y oxidación por ultravioleta/ozono, catalogados como POA. Estos procesos son una alternativa debido a que generan radicales hidroxilo $\cdot \mathrm{OH}$, caracterizados por su alto poder oxidante. $\mathrm{La}$ electrooxidación se puede declarar como la más conveniente para la degradación de edulcorantes artificiales como el acesulfamo de potasio, por ejemplo, debido a la menor cantidad de componentes requeridos para su ejecución y, al mismo tiempo, se logra una eficiencia de remoción similar, o incluso mayor, comparada con otros POA. Igualmente, se documentaron los resultados y méritos relativos a la aplicación de estos al agua o matrices acuosas, así como las oportunidades para investigación futura.

PALABRAS CLAVE: Edulcorante artificial; POA; electrooxidación; luz UV; radical hidroxilo.

\section{ABSTRACT}

The objective of this review is to present recent research on the removal of some sweeteners-recognized as emerging contaminants-from water or aqueous matrices, using the Advanced Oxidation Processes (AOPs). In an attempt to evade the consumption of caloric sweeteners that has caused health problems, humans consume artificial sweeteners that are practically not metabolized after ingestion, thus integrating into wastewater and even resisting total degradation in plants conventional water treatment. Therefore, electro-oxidation, electro-Fenton, photo-Fenton, heterogeneous photocatalysis, ultraviolet / peroxide oxidation and ultraviolet / ozone oxidation cataloged as AOPs were reviewed in databases. These processes are an alternative because they generate hydroxyl radicals $\cdot \mathrm{OH}$ characterized by their high oxidizing power. Electro-oxidation can be declared as the most convenient for the degradation of artificial sweeteners such as potassium acesulfame, for example, due to the lower amount of components required for its execution and at the same time the achievement of a similar removal efficiency, or even greater, compared to other AOPs. Likewise, the results and merits related to their application to water or aqueous matrices were documented, as well as the opportunities for future research.

KEYWORDS: Artificial sweetener; AOPs; electro-oxidation; UV light; hydroxyl radical.

Correspondencia:

DESTINATARIO: Humberto Rubí-Juárez

INSTITUCIÓN: Universidad Autónoma de Ciudad Juárez

DIRECCIÓN: Avenida del Charro 450 norte, col. Partido Romero, C. P. 32310, Ciudad Juárez, Chihuahua, México. CORREO ELECTRÓNICO: humberto.rubi@uacj.mx
Fecha de recepción: 14 de enero de 2020

Fecha de aceptación: 17 de febrero de 2020

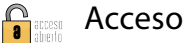

UACJ abierto
Revisión

por pares 


\section{INTRODUCCIÓN}

La propensión que predomina hacia el consumo de alimentos dulces se ha imputado a la tendencia que tienen los seres humanos a experimentar sensaciones estimulantes ${ }^{[1]}$. El elevado consumo de azúcar se ha relacionado desde hace muchos años con enfermedades cardiovasculares, obesidad, diabetes mellitus, incidencia de caries y otros trastornos metabólicos ${ }^{[2]}$. La consecuencia fue que la industria alimentaria desarroló alternativas para satisfacer el deseo por los sabores dulces: los denominados sustitutos del azúcar, edulcorantes no nutritivos o edulcorantes artificiales ${ }^{[3]}$.

Desde hace varias décadas se han formulado varios edulcorantes ${ }^{[4]}$, por ejemplo, el aspartamo en $1965^{[5]}$. Sin embargo, se han clasificado en dos generaciones: la primera incluyó aspartamo, 200 veces más dulce que la sacarosa, sacarina y ciclamato, mientras que la segunda abarcó acesulfamo-K, 300 veces más dulce que la sacarosa, alitamo, neotamo y sucralosa ${ }^{[6]}$. Las estructuras químicas de los compuestos señalados, se muestran en la Figura 1.
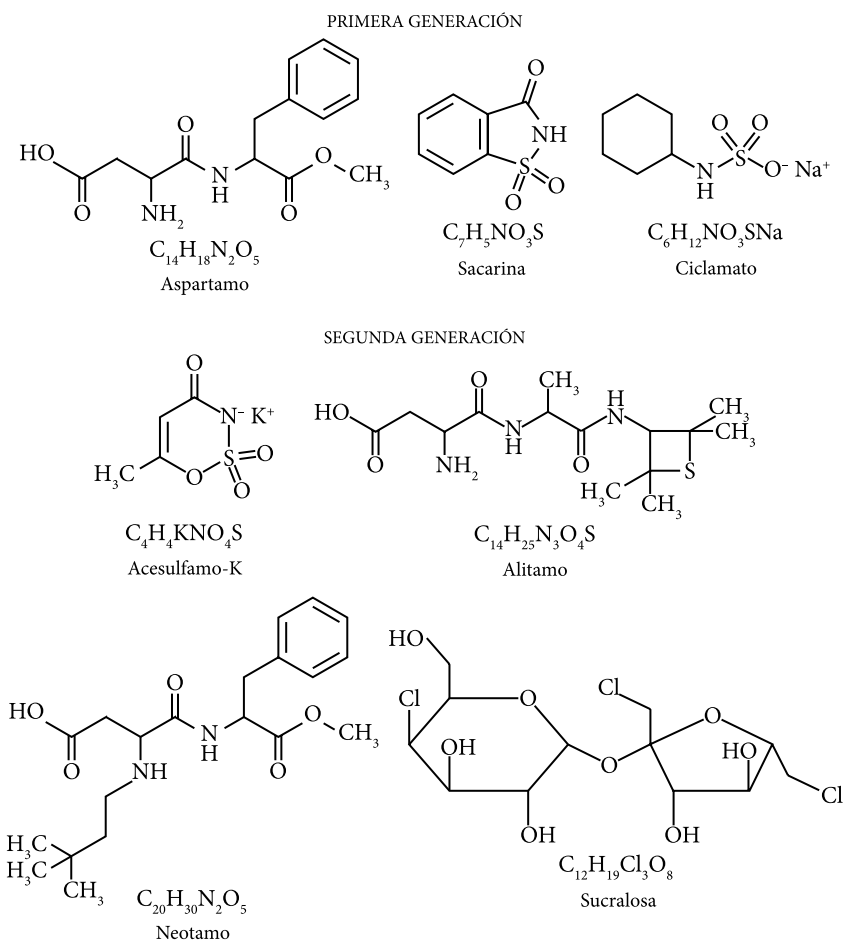

Figura 1. Estructuras químicas de algunos edulcorantes.

Los edulcorantes no son metabolizados convencionalmente como los carbohidratos ${ }^{[7]}$, lo cual, aunado a la alta ingesta según las relaciones de producción-de- manda ${ }^{[8]}$, ha provocado la proliferación de diversos compuestos como acesulfamo, sacarina, sucralosa, aspartamo y ciclamato en el agua residual, subterránea, superficial y potable ${ }^{[9]}$. Una vez que los edulcorantes ingresan a los cuerpos de agua, los subproductos obtenidos por fotodegradación son más tóxicos que los compuestos originales ${ }^{[10]}$.

En ese contexto, los edulcorantes artificiales, debido a su relativamente reciente y frecuente detección en numerosos ambientes, han sido considerados como contaminantes emergentes ${ }^{[11]}$, especialmente la sacarina, el ciclamato, el acesulfamo y la sucralosa ${ }^{[12]}$. Por consiguiente, la remoción de esos contaminantes del agua se proyecta como una situación de extrema relevancia en la que los Procesos de Oxidación Avanzada (POA) son una alternativa interesante ${ }^{[7]}$.

Los POA son procesos que invariablemente requieren la formación de radicales hidroxilo $\cdot \mathrm{OH}$. Últimamente se ha tomado en cuenta a los radicales sulfato $\mathrm{SO}_{4}^{-{ }^{-}}{ }^{[13]}$, en solución acuosa en medio heterogéneo u homogéneo, usando energía u omitiéndola. Originalmente los POA se distinguieron porque no participaban oxidantes habituales como cloro, dióxido de cloro ${ }^{[14]}$, y posteriormente ozono ${ }^{[13]}$, ya que todos ellos tienen un menor potencial de oxidación que el $\cdot \mathrm{OH}(2.8 \mathrm{eV})^{[15,16]}$.

Por tanto, esta revisión presenta investigaciones recientes que se han realizado sobre la remoción de algunos edulcorantes, reconocidos como contaminantes emergentes, del agua o matrices acuosas con base en los POA. Particularmente, se analizan: 1) electrooxidación, 2) electro-Fenton, 3) foto-Fenton, 4) fotocatálisis heterogénea, 5) ultravioleta/peróxido y 6) ultravioleta/ ozono, que son los que fueron encontrados en las bases de datos ScienceDirect, Springer Link, Taylor \& Francis, Wiley y rsc.org.

\section{PROCESOS DE OXIDACIÓN AVANZADA}

\section{A. Electrooxidación}

La electrooxidación se lleva a cabo con electrodos sumergidos en el agua, los cuales se conectan a una fuente de alimentación que suministra el voltaje para producir corriente eléctrica que pasa a través de ellos. En el arreglo, uno o varios electrodos actúan como ánodo/s y otro/s como cátodo/s. El propósito de la electrooxidación es el aprovechamiento de las reacciones redox 
que tienen lugar, oxidación en el ánodo y reducción en el cátodo, para la remoción de contaminantes ${ }^{[17]} \mathrm{de}$ carácter comúnmente orgánico ${ }^{[18]}$. La electrooxidación se realiza de dos maneras:

1) Oxidación directa. Los radicales $\cdot \mathrm{OH}$ que son producidos en la superficie del ánodo se encuentran disponibles para la oxidación de compuestos ${ }^{[19]}$.

2) Oxidación indirecta. Las especies oxidantes son electrogeneradas en la superficie del ánodo, por ejemplo, hipoclorito $\left(\mathrm{OCl}^{-}\right)$, ácido hipocloroso ( $\mathrm{HOCl})$, peroxidisulfato $\left(\mathrm{S}_{2} \mathrm{O}_{8}^{2-}\right)$ y peróxido de hidrógeno $\left(\mathrm{H}_{2} \mathrm{O}_{2}\right){ }^{[20]}$.

Los materiales electródicos típicos para el tratamiento de agua por electrooxidación, conocida como oxidación electroquímica e incluso oxidación anódica, son dióxido de estaño $\left(\mathrm{SnO}_{2}\right)$, plomo, dióxido de plomo $\left(\mathrm{PbO}_{2}\right)$, ánodos dimensionalmente estables y Diamante Dopado con Boro (DDB) ${ }^{[19,20]}$.

Punturat y Huang ${ }^{[21]}$ llevaron a cabo la electrooxidación de acesulfamo de potasio (ACE-K) en solución acuosa, así como en agua residual doméstica sin tratar, antes y después de un tratamiento biológico. Las muestras de agua fueron colectadas en el sur de Taiwán. En el ánodo, los electrodos que se utilizaron fueron Diamante Dopado con Boro, $\mathrm{Pt}$ o $\mathrm{PbO}_{2}$, en conjunto con el acero inoxidable en el cátodo. Se evaluaron variables como densidad de corriente (j), área anódica, temperatura y matriz de agua residual. Inicialmente, con una $j=0.1 \mathrm{~A} / \mathrm{cm}^{2}, 2 \mathrm{~cm}^{2}$ de área, $100 \mathrm{mg} / \mathrm{L}$ de ACE-K y $25{ }^{\circ} \mathrm{C}$, la degradación alcanzó el $72 \%$ después de 120 minutos con $\mathrm{Pt}$, y $100 \%$ transcurridos 90 y 30 minutos con $\mathrm{PbO}_{2}$ y DDB, respectivamente.

El DDB también degrada completamente el ACE-K a $0.05 \mathrm{~A} / \mathrm{cm}^{2}$, pero requirió de 90 minutos. Por lo tanto, los electrodos de DDB se eligieron para la exploración de las variables remanentes. El incremento de área anódica de $2 \mathrm{~cm}^{2}$ a $4 \mathrm{~cm}^{2}$ mejoró la degradación de ACE-K a una concentración de $200 \mathrm{mg} / \mathrm{L}$ y $25^{\circ} \mathrm{C}$, $100 \%$ al término de 50 minutos, disminuyendo hasta 20 minutos con el doble de área. El efecto de la temperatura se detectó a través de la remoción de Carbono Orgánico Total (COT), lo que produjo $100 \%$ de remoción a $50{ }^{\circ} \mathrm{C}$ y 40 minutos, mientras que $84 \%$ se obtuvo en 120 minutos y $25^{\circ} \mathrm{C}$.
Todas las soluciones conformadas con ACE-K, $1 \mathrm{M}$ de $\mathrm{Na}_{2} \mathrm{SO}_{4}$ y las de agua residual, alcanzaron degradación completa en menos de 30 minutos. La electrooxidación constituyó un método para la remoción de ACE-K y COT de las aguas residuales.

\section{B. Fenton y variantes}

\section{B.1. Fenton}

El proceso requiere, según el escenario básico, ion ferroso $\left(\mathrm{Fe}^{2+}\right)$ y $\mathrm{H}_{2} \mathrm{O}_{2}$ para formar radicales $\cdot \mathrm{OH}$ que provoca la oxidación de materia orgánica. Ya ha sido propuesto en Walling ${ }^{[22]}$ un modelo detallado de reacciones en el se supone la generación de tres tipos de radicales que experimentan oxidación, dimerizan o son reducidos. Expresado en forma simplificada para incorporar el radical perhidroxilo $\left(\mathrm{HO}_{2}^{*}{ }^{[23]}\right.$, se tiene:

$$
\begin{gathered}
\mathrm{Fe}^{2+}+\mathrm{H}_{2} \mathrm{O}_{2} \longrightarrow \mathrm{Fe}^{3+}+\mathrm{HO}^{-}+\mathrm{HO}^{\cdot} \\
\mathrm{Fe}^{3+}+\mathrm{H}_{2} \mathrm{O}_{2} \longrightarrow \mathrm{Fe}^{2+}+\mathrm{HO}_{2}^{-}+\mathrm{H}^{+} \\
\mathrm{Fe}^{3+}+\mathrm{HO}_{2}^{-} \longrightarrow \mathrm{Fe}^{2+}+\mathrm{O}_{2}+\mathrm{H}^{+} \\
\mathrm{HO}+\mathrm{H}_{2} \mathrm{O}_{2} \quad \mathrm{HO}_{2}^{-}+\mathrm{H}_{2} \mathrm{O}
\end{gathered}
$$

El pH óptimo oscila entre 2 y 4 , por lo que es necesario ajustarlo dentro de ese rango. Un valor mayor a 4 propiciaría la precipitación del hierro como hidróxido férrico, separándolo del medio acuoso. $\mathrm{El} \mathrm{Fe}(\mathrm{OH})_{3}$ reaccionaría con el $\mathrm{H}_{2} \mathrm{O}_{2}$ sin que se forme el radical $\cdot \mathrm{OH}{ }^{[24]}$. Las variables que se consideran para evaluar el efecto que tienen en el tratamiento de agua residual son: 1) $\mathrm{pH}$ de operación, 2) cantidad de iones ferrosos, 3) concentración de $\left.\mathrm{H}_{2} \mathrm{O}_{2}, 4\right)$ concentración inicial del contaminante, 5) tipo de solución amortiguadora utilizada para ajuste de $\mathrm{pH}$ y 6) temperatura de operación ${ }^{[25]}$.

\section{B.2. Electro-Fenton}

El proceso electro-Fenton requiere del catión $\mathrm{Fe}^{2+}$ y de peróxido de hidrógeno, ya sea que se generen dentro de la celda electroquímica o bien que desde el exterior se añadan a la misma, con el propósito de producir radicales $\cdot \mathrm{OH}$. Las tres combinaciones para llevarlo a cabo son: 1) adición de $\mathrm{Fe}^{2+}$ con generación electroquímica de $\mathrm{H}_{2} \mathrm{O}_{2}$, 2) adición de $\mathrm{H}_{2} \mathrm{O}_{2}$ con $\mathrm{Fe}^{2+}$ electrogenerado y 3 ) electrogeneración de $\mathrm{H}_{2} \mathrm{O}_{2}$ y $\mathrm{Fe}^{2+}$. El mecanismo más comúnmente explicado contempla 
la generación de $\mathrm{H}_{2} \mathrm{O}_{2}$ a través de la reducción de oxígeno en la superficie del cátodo ${ }^{[26]}$,

$$
\mathrm{O}_{2}+2 \mathrm{H}^{+}+2 e^{-} \longrightarrow \mathrm{H}_{2} \mathrm{O}_{2}
$$

y la adición o producción de $\mathrm{Fe}^{2+}$ desde el ánodo ${ }^{[27]}$,

$$
\mathrm{Fe}^{0} \longrightarrow \mathrm{Fe}^{2+}+e^{-}
$$

que conduce a la generación de radicales $\cdot \mathrm{OH}$ activos. Adicionalmente, el $\mathrm{Fe}^{2+}$ puede ser regenerado por medio de la reducción de $\mathrm{Fe}^{3+}$ en el cátodo ${ }^{[28]}$,

$$
\mathrm{Fe}^{3+} e^{-} \longrightarrow \mathrm{Fe}^{2+}
$$

creando también en el ánodo radicales $\cdot \mathrm{OH}$ con la oxidación del $\mathrm{H}_{2} \mathrm{O}{ }^{[27]}$.

$$
\mathrm{H}_{2} \mathrm{O} \longrightarrow \mathrm{HO}^{\cdot}+\mathrm{H}^{+}+e^{-}
$$

Los factores que se ha reconocido que influyen en electro-Fenton son $\left.{ }^{[29]}: 1\right)$ concentración de la relación $\left.\mathrm{Fe}^{2+} / \mathrm{Fe}^{3+}, 2\right)$ concentración de $\mathrm{H}_{2} \mathrm{O}_{2}, 3$ ) concentración inicial de contaminantes, 4) $\mathrm{pH}, 5)$ electrolito como $\mathrm{Na}_{2} \mathrm{SO}_{4}, \mathrm{KCl}, \mathrm{NaCl}, \mathrm{NaNO}_{3}$ o $\mathrm{NaClO}_{4}$ en el rango de 50 a $100 \mathrm{mM}, 6)$ velocidad de rociado de oxígeno, 7) modo de alimentación de oxígeno, 8) densidad de corriente, 9) temperatura de operación, 10) materiales electródicos y 11) distancia entre electrodos.

Lin et al. ${ }^{[30]}$ investigaron la degradación de aspartamo (ASP) en solución acuosa. Los ensayos se llevaron a cabo en una celda de vidrio cilíndrica que tenía acoplado fieltro de carbono en todo el interior de la pared, actuando como cátodo. El ánodo se situó en el centro como una malla de Pt o una película de DDB. El suministro de $\mathrm{H}_{2} \mathrm{O}_{2}$ se realizó por medio de la electrogeneración con oxígeno, burbujeando hacia la solución un flujo de aire a razón de $0.5 \mathrm{~L} / \mathrm{min}$. Las concentraciones fueron de $0.2 \mathrm{mM}$ el ASP y de $50 \mathrm{mM}$ el $\mathrm{Na}_{2} \mathrm{SO}_{4}$ que se ocupó como electrolito de soporte. El catalizador, ion ferroso, se añadió previamente y el $\mathrm{pH}$ se ajustó a alrededor de 3 .

Cuando la intensidad de corriente (I) fue de $200 \mathrm{~mA}$ y la concentración de $\mathrm{Fe}^{2+}$ de $0.2 \mathrm{mM}$, todo el ASP se degradó en 20 minutos o en 15 minutos con el ánodo de Pt o DDB, respectivamente. Asimismo, el incremento de concentración de $\mathrm{Fe}^{2+}$ hasta $0.5 \mathrm{mM}$ disminuyó la eficiencia de remoción por la competencia que ocurre por los radicales $\cdot \mathrm{OH}$. La remoción de COT a $200 \mathrm{~mA}$ y 360 minutos de electrólisis se ubicó en 81.7 y $97.5 \%$ usando Pt y DDB, respectivamente. La variación de la corriente con las dos configuraciones de electrodos, $\mathrm{Pt}$ o DDB con fieltro de carbono, reveló un efecto sobre la constante cinética $(k)$ de un modelo de pseudo primer orden que propusieron estos autores. El incremento de 50 a $300 \mathrm{~mA}$ aumentó $k$ de 0.14 a $0.34 \mathrm{~min}^{-1}$ para el sistema $\mathrm{Pt} /$ carbono. La corriente óptima en el arreglo de $\mathrm{DDB} /$ carbono fue de $200 \mathrm{~mA}$, mejorando el consumo de energía. El DDB consiguió la mineralización más rápido cuando se comparó con el Pt.

Lin et al. ${ }^{[31]}$ evaluaron la degradación de sucralosa (SUC) en solución acuosa. Las dos variables tomadas en cuenta fueron la concentración del catalizador $\left(\mathrm{Fe}^{2+}\right)$ y la corriente aplicada. Se utilizó una configuración de celda cilíndrica de vidrio con ánodo de $\mathrm{Pt}$ o DDB situado en el centro. El cátodo fue una pieza de $105 \mathrm{~cm}^{2}$ de fieltro de carbono que rodeaba toda la pared interior de la celda. En este se generó el $\mathrm{H}_{2} \mathrm{O}_{2}$ a través de la reducción de $\mathrm{O}_{2}$. La dotación se garantizó burbujeando aire comprimido a razón de $0.5 \mathrm{~L} /$ minuto y se determinó en los experimentos una concentración de $0.2 \mathrm{mM}$ y $50 \mathrm{mM}$, concerniente a SUC y $\mathrm{Na}_{2} \mathrm{SO}_{4}$, respectivamente. Previo a la electrólisis, el ion ferroso se agregó y el pH se ajustó a 3.

Los autores encontraron que la concentración óptima de $\mathrm{Fe}^{2+}$ fue de $0.2 \mathrm{mM}$ para los ánodos de Pt y DDB. Bajo las condiciones de $0.2 \mathrm{mM}$ y $200 \mathrm{~mA}$, establecidas como óptimas, el DDB removió $96.1 \%$ del COT inicial con 2 horas de tratamiento. El mismo porcentaje requirió 4 horas para Pt. La exploración del efecto de la corriente osciló entre 100-500 mA, dejando el $\mathrm{Fe}^{2+}$ a 0.2 mM. La remoción de COT fue de $69.7 \%$ con 6 horas de electrólisis a $100 \mathrm{~mA}$, pero al incrementar la corriente a $200 \mathrm{~mA}$ el COT se reduce hasta $94.1 \%$ en 4 horas. En los procesos de electro-Fenton, ambos electrodos Pt y DDB, aplicado a una relación equimolar $0.2 \mathrm{mM}$ $\mathrm{SUC} / \mathrm{Fe}^{2+}$, empleando $200 \mathrm{~mA}$ de corriente y $\mathrm{pH}$ igual a 3, se observó la presencia de ácido oxálico, pirúvico, fórmico y glicólico.

Lin et al. ${ }^{[32]}$ estudiaron la degradación de sacarina (SAC) por medio del proceso electro-Fenton en medio acuoso. Se analizaron el comportamiento de la cinética de mineralización y el de la mineralización. Las pruebas se realizaron en una celda de vidrio cilíndrica con capacidad de $250 \mathrm{~mL}$ para reservar $220 \mathrm{~mL}$ de 
solución. Los ánodos colocados en el centro de la celda fueron DDB, Pt o una mezcla de óxidos metálicos consistentes en $\mathrm{Ti} / \mathrm{RuO}_{2}-\mathrm{IrO}_{2}$, comprendidos dentro de un grupo general de materiales anódicos conocido como ánodos dimensionalmente estables (ADS, por sus siglas en inglés) ${ }^{[33]}$. El cátodo de fieltro de carbono que cubría el interior del cilindro también rodeaba al ánodo. Elementos adicionales constaron de $50 \mathrm{mM}$ de $\mathrm{Na}_{2} \mathrm{SO}_{4}$, catalizador $\mathrm{Fe}^{2+}$ y $\mathrm{pH}$ igual a 3. Una cinética de pseudo primer orden fue delineada con valores de constante de velocidad aparente $\left(k_{\mathrm{ap}}\right) 0.18,0.19$, y 0.21 min $^{-1}$ para ADS, Pt y DDB, respectivamente.

En cuanto a los porcentajes de remoción de COT, se lograron 55.8, 76.1 y $96.2 \%$ en ese mismo orden anódico. Reconociendo la superioridad del DDB, el efecto de la concentración de $\mathrm{Fe}^{2+}$ con ese ánodo exhibió que 0.2 $\mathrm{mM}$ del catalizador maximizaron la remoción de SAC. Asimismo, la $k_{\text {ap }}$ para la oxidación de SAC aumentó de 0.09 hasta $0.19 \mathrm{~min}^{-1}$ cuando la corriente aplicada se incrementó de 50 a $200 \mathrm{~mA}$. Los valores superiores, por ejemplo, de $500 \mathrm{~mA}$, provocan una disminución concertándose en un valor de $k_{\text {ap }}$ igual a $0.16 \mathrm{~min}^{-1}$ que atribuyeron a reacciones secundarias que consumen radicales $\cdot \mathrm{OH}$ o la recombinación de estos con el $\mathrm{DDB}$, representado como $\mathrm{BBD}(\cdot \mathrm{OH})$. Electro-Fenton demostró que la SAC se puede oxidar en aproximadamente 25 minutos en el escenario determinado con la aparición de ácidos carboxílicos de cadena corta como productos intermediarios.

Lin et al. ${ }^{[34]}$ investigaron la cinética de degradación y mineralización de SAC con electro-Fenton. Las variables examinadas fueron corriente aplicada, catalizador $\mathrm{Fe}^{2+}$, materiales anódicos y electrolito de soporte. Las características de la celda utilizada, incluyendo ánodos, cátodo, concentración de electrolito y $\mathrm{pH}$, ya han sido descritas en los párrafos anteriores ${ }^{[30,31,32]}$. Complementariamente, se destacó el valor de $\mathrm{pH}$ de 2.8 , que permaneció al final del tratamiento. Las diferentes concentraciones de electrolitos, como $50 \mathrm{mM} \mathrm{Na}_{2} \mathrm{SO}_{4}$, $100 \mathrm{mM} \mathrm{NaNO}_{3}$ y $100 \mathrm{mM} \mathrm{NaCl}$, produjeron tendencias de decaimiento de tipo exponencial. Entonces, fue posible ajustar un modelo cinético de primer orden.

Los tres ánodos, $\mathrm{Pt}$, DDB y ADS, alcanzaron $100 \%$ de remoción casi al finalizar un periodo de electrólisis de 25 minutos en presencia de $\mathrm{Na}_{2} \mathrm{SO}_{4}$ y $\mathrm{NaNO}_{3}$. Por otra parte, solo se abatió un $50 \%$ de $\mathrm{SAC}$ con $\mathrm{NaCl}$ en 30 minutos, debido a la generación de ácido hipocloroso $(\mathrm{HClO})$ con menor poder oxidante que el $\cdot \mathrm{OH}$. Puesto que la velocidad de degradación con $\mathrm{Na}_{2} \mathrm{SO}_{4}$ fue un poco mayor que con $\mathrm{NaNO}_{3}$, por ejemplo, una $k_{\text {ap }}$ de $0.18 \mathrm{~min}^{-1}$ superior a $0.17 \mathrm{~min}^{-1}$, si el ADS es usado, el $\mathrm{Na}_{2} \mathrm{SO}_{4}$ fue elegible en lo subsecuente. La concentración de $0.2 \mathrm{mM}$ de $\mathrm{Fe}^{2+}, 50 \mathrm{mM}$ de $\mathrm{Na}_{2} \mathrm{SO}_{4}$, una corriente de $200 \mathrm{~mA}$ y un $\mathrm{pH}$ de 3, condujo a una remoción de COT de 55.8, 76.1 y 96.2\% para DSA, Pt y DDB, respectivamente. La velocidad de oxidación fue mejorada cuando la concentración de $\mathrm{Fe}^{2+}$ aumentó de 0.05 a $0.2 \mathrm{mM}$ para el ánodo de Pt y DDB. El incremento adicional a $0.5 \mathrm{mM}$ disminuyó la velocidad de degradación debido a reacciones indeseables del $\mathrm{Fe}^{2+}$ con el $\cdot \mathrm{OH}$. El tiempo para la desaparición completa de SAC cambió de 30 minutos para $100 \mathrm{~mA}$ a 25, 20 y 15 minutos para 200, 300 y $500 \mathrm{~mA}$ con el electrodo de Pt. Por el contrario, el DDB disminuyó la velocidad con la corriente de $500 \mathrm{~mA}$ imputable a una reacción de competencia en la reducción de $\mathrm{O}_{2}$ que forma $\mathrm{H}_{2} \mathrm{O}$. La mejor mineralización del DDB contra el Pt fue aludida a un poder de oxidación superior.

\section{B.3. Foto-Fenton}

En condiciones ácidas, con intervalo de $\mathrm{pH}$ de 2.8-3.5, el $\mathrm{Fe}^{3+}$ prevalece en la forma dominante $\mathrm{Fe}(\mathrm{OH})^{2+}$, según el diagrama de especiación ${ }^{[35]}$. La irradiación con luz UV a una longitud de onda menor a $580 \mathrm{~nm}$ genera radicales hidroxilo ${ }^{[36]}$ :

$$
\mathrm{Fe}(\mathrm{OH})^{2+}+h v \longrightarrow \mathrm{Fe}^{2+}+\mathrm{HO}^{\cdot}
$$

Adicionalmente, la fotólisis del $\mathrm{H}_{2} \mathrm{O}_{2}$ ocurre a longitudes de onda menores a $310 \mathrm{~nm}$ :

$$
\mathrm{H}_{2} \mathrm{O}_{2}+h v \longrightarrow 2 \mathrm{HO}
$$

Por lo tanto, se tendrán radicales extra disponibles para la oxidación. En particular, la secuencia posterior para que suceda la mineralización de la materia orgánica (RH) se presenta como sigue ${ }^{[37]}$ :

$$
\begin{gathered}
\mathrm{HO}^{\bullet}+\mathrm{RH} \longrightarrow \mathrm{R}^{\bullet}+\mathrm{H}_{2} \mathrm{O} \\
\mathrm{R}^{\bullet}+\mathrm{O}_{2} \longrightarrow \mathrm{RO}_{2}^{*}
\end{gathered}
$$

El producto de la última reacción tiene la capacidad de iniciar reacciones en cadena para la destrucción oxidativa. 
Kattel et al. ${ }^{[38]}$ degradaron acesulfamo (ACE) con UVA y siete esquemas que involucran reactivos UVA/ $\mathrm{H}_{2} \mathrm{O}_{2}, \mathrm{UVA} / \mathrm{Fe}^{2+}, \mathrm{UVA} / \mathrm{S}_{2} \mathrm{O}_{8}^{2-}, \mathrm{H}_{2} \mathrm{O}_{2} / \mathrm{Fe}^{2+}, \mathrm{S}_{2} \mathrm{O}_{8}^{2-} / \mathrm{Fe}^{2+}$, UVA $/ \mathrm{H}_{2} \mathrm{O}_{2} / \mathrm{Fe}^{2+}$ y UVA $/ \mathrm{S}_{2} \mathrm{O}_{8}^{2-} / \mathrm{Fe}^{2+}$. La fase acuosa con ACE fue agua ultrapura (AU), agua subterránea (AS) y efluente de tratamiento secundario (AR), ambas de Estonia. Las concentraciones de oxidante e ion ferroso y valores de $\mathrm{pH}$ fueron varias. La fuente de UVA consistió de una lámpara de baja presión de mercurio localizada en el interior de un tubo de cuarzo que se encontraba dentro de un reactor cilíndrico de vidrio. El líquido se mantuvo agitado a una velocidad de 400 $\mathrm{rpm}$, con una concentración de ACE igual a $75 \mu \mathrm{M}$. La reacción requirió $\mathrm{Na}_{2} \mathrm{~S}_{2} \mathrm{O}_{8}$ o $\mathrm{H}_{2} \mathrm{O}_{2}$ y $\mathrm{FeSO}_{2} \cdot 7 \mathrm{H}_{2} \mathrm{O}$, según fuera necesario. La degradación de ACE en AU para la concentración inicial de oxidante de $150 \mu \mathrm{M}$ hasta $750 \mu \mathrm{M}$ fue más rápida con $\mathrm{UVA} / \mathrm{H} 2 \mathrm{O} 2 / \mathrm{Fe}^{2+}$, entre 10-30 minutos, comparada con $\mathrm{UVA} / \mathrm{S}_{2} \mathrm{O}_{8}^{2-} / \mathrm{Fe}^{2+}$ en el intervalo de 30-45 minutos.

La remoción de COT fue de aproximadamente $80 \%$ después de 120 minutos con $1500 \mu \mathrm{M}$ de oxidante para ambos planteamientos, también en AU. La degradación del ACE en las matrices acuosas, AS y AR, fue notoriamente más baja. Considerando una relación molar de $1 / 10 / 1$ en UVA $/ \mathrm{H}_{2} \mathrm{O}_{2} / \mathrm{Fe}^{2+}$ y UVA $/ \mathrm{S}_{2} \mathrm{O}_{8}^{2-} / \mathrm{Fe}^{2+}$, a un $\mathrm{pH}$ de 7.75, la eliminación de ACE fue de 6.5 y 5.5\% en AS, respectivamente. En AR hubo un caso similar al de AS, puesto que la remoción alcanzó 7.9\% y 4.8\% correspondientes a UVA $/ \mathrm{H}_{2} \mathrm{O}_{2} / \mathrm{Fe}^{2+}$ y UVA $/ \mathrm{S}_{2} \mathrm{O}_{8}^{2-} / \mathrm{Fe}^{2+}$ transcurridos 120 minutos. Según la literatura y conocimiento disponible de los autores, este fue el primer estudio aplicado al tratamiento de ACE en diferentes matrices acuosas vía foto-Fenton.

\section{Fotocatálisis heterogénea}

La fotocatálisis se inicia por la adsorción de un fotón con energía igual o mayor que la banda prohibida de un semiconductor para producir pares electrón-hueco. La consecuencia originada es la existencia de electrones en la banda de conducción y de huecos en la de valencia, en la que la falta de electrones asocia una carga contraria a la del electrón, a saber, positiva ${ }^{[39]}$. En la superficie externa del semiconductor, $\mathrm{TiO}_{2}$ por ejemplo, los electrones y huecos intervienen en las reacciones redox de alguna especie adsorbida como agua, ion hidróxido, compuestos orgánicos u oxígeno. Los huecos producen, a través de la oxidación, al radical $\bullet \mathrm{OH}$ a partir de $\mathrm{H}_{2} \mathrm{O}$ o iones $\mathrm{OH}^{-}{ }^{[40]}$. Las reacciones son:

$$
\begin{gathered}
\mathrm{TiO}_{2}\left(h_{v b}^{+}\right)+\mathrm{H}_{2} \mathrm{O}_{a d s} \longrightarrow \mathrm{TiO}_{2}+\mathrm{OH}_{a d s}^{\mathrm{o}}+\mathrm{H} \\
\mathrm{TiO}_{2}\left(h_{v b}^{+}\right)+\mathrm{OH}_{a d s}^{-} \longrightarrow \mathrm{TiO}_{2}+\mathrm{OH}_{a d s}^{\mathrm{o}}
\end{gathered}
$$

donde $h_{v b}^{+}$es el hueco en la banda de valencia ${ }^{[41]}$. La aplicación de la fotocatálisis se originó hacia 1972, cuando la hidrólisis fotocatalítica del agua se llevó a cabo usando electrodos de $\mathrm{TiO}_{2}$ y, entonces, proliferó la investigación en relación a la fotocatálisis heterogénea ${ }^{[42]}$.

Calza et al. ${ }^{[43]}$ sintetizaron diferentes catalizadores para estimar su desempeño fotocatalítico, sometido a luz solar y visible, para abatir el ACE-K en agua ultrapura y de río, conteniendo $4.8 \mathrm{mg} / \mathrm{L}$ de materia orgánica disuelta. El proceso fue hidrotérmico, notable por su accesibilidad y bajo costo, para dopar $\mathrm{ZnO}$ con una carga de cerio de 0.5 y $1 \%$. Las nanoestructuras obtenidas a bajas temperaturas las compararon contra $\mathrm{TiO}_{2}$ P25 comercial que posee una relación de fases aproximada de 3:1 de anatasa y rutilo ${ }^{[44]}$. El ZnO sin dopar se formuló partiendo de una solución de acetato de zinc a la que se le añadió $\mathrm{NaOH}$ hasta llegar a un $\mathrm{pH}$ entre 10 y 11. La solución se transfirió a un autoclave de acero inoxidable a $175^{\circ} \mathrm{C}$, permaneciendo toda la noche.

La muestra de $\mathrm{ZnO}$ dopada con cerio fue preparada agregando a la solución inicial dos diferentes sales precursoras: $\mathrm{CeCl}_{3} \cdot 7 \mathrm{H}_{2} \mathrm{O}$ y Ce $\left(\mathrm{SO}_{4}\right)_{2}$, creando $\mathrm{Ce} 05 \mathrm{Cl}$, $\mathrm{Ce} 1 \mathrm{Cl}, \mathrm{Ce} 05 \mathrm{~S}$ y $\mathrm{Ce} 1 \mathrm{~S}$ relacionadas con cloruro y sulfato de cerio en porcentaje 0.5 y $1 \%$. La concentración inicial de ACE-K fue de $20 \mathrm{mg} / \mathrm{L}$, con cargas de catalizador de $100 \mathrm{mg} / \mathrm{L}$ dentro del reactor cilíndrico de cuarzo. La irradiación, a una distancia de $18 \mathrm{~cm}$, utilizó una lámpara de arco de xenón y filtros que restringen la transmisión de longitudes de onda menores a 290 $\mathrm{nm}$, simulando luz solar. La intensidad de radiación se mantuvo en $750 \mathrm{~W} / \mathrm{m}^{2}$. Un filtro se usó para establecer luz visible con longitud de onda $(\lambda)$ mayor a $430 \mathrm{~nm}$. La temperatura de la cámara y las muestras de agua se controló con circuitos de enfriamiento. Previo a la irradiación se dejó que el catalizador alcanzara el equilibrio de adsorción en la suspensión, permaneciendo en un sitio oscuro por 60 minutos. Las muestras se filtraron con tamaño de poro de $0.45 \mu \mathrm{m}$ antes del análisis. La fotólisis directa se ejecutó como blanco para calcular su contribución en la degradación. En agua ultrapura y de río, el Ce1S logró la mejor remoción: alrededor de $68 \%$ y $42 \%$, respectivamente, independientemente de la fuente de luz solar o visible. 
Ghosh et al. ${ }^{[45]}$ exploraron la degradación de ACE-K con incidencia de luz solar simulada, en el intervalo $\lambda$ de 300 a $650 \mathrm{~nm}$, y el fotocatalizador semiconductor $\mathrm{TiO}_{2}$. Las concentraciones iniciales de ACE-K, dosis de fotocatalizador e intensidad de luz solar fueron variadas por los investigadores. Adicionalmente, sin luz se realizaron experimentos de adsorción para determinar el tiempo de equilibrio y el modelo de isoterma de equilibrio de adsorción, que mejor ajustó los datos. $\mathrm{El} \mathrm{ZnO}$ sirvió para efectuar una comparación de actividad fotocatalítica respecto a $\mathrm{TiO}_{2}$. El simulador solar tenía una lámpara de arco de xenón de $1000 \mathrm{~W}$ capaz de ocasionar una irradiación de $100 \mathrm{~mW} / \mathrm{cm}^{2}$ a máxima potencia.

Las reacciones fotocatalíticas ocurrieron al interior de un reactor de vidrio Pyrex, con dimensiones de $11 \mathrm{~cm}$ de diámetro y $6.3 \mathrm{~cm}$ de alto, con una ventana plana en la zona superior para la fuente de luz situada a $39 \mathrm{~cm}$. La adsorción consideró cinco concentraciones iniciales, comenzando desde $10 \mathrm{mg} / \mathrm{L}$ para terminar en 50 mg/L, combinándolos con los valores de $\mathrm{pH} \mathrm{6,} 7$ y 8 . Por su parte, seis diferentes dosis de $\mathrm{TiO}_{2}$ se acoplaron con esas mismas concentraciones iniciales de ACE-K. Igualmente, el efecto de la intensidad de la luz contempló cinco intensidades desde 20 a $100 \mathrm{~mW} / \mathrm{cm}^{2}$ para las concentraciones de edulcorante mencionadas.

Los estudios de adsorción mostraron que se alcanzó el equilibrio después de una hora, independientemente de la concentración inicial de ACE-K. El coeficiente de adsorción a $\mathrm{pH} 6$ fue ligeramente mayor que a $7 \mathrm{y}$ 8 , lo que se relacionó con la superficie del $\mathrm{TiO}_{2}$ cargada positivamente y el ACE-K disociado según el $\mathrm{pKa}$ bajo. El modelo de Freundlich presentó un mejor ajuste en comparación con Langmuir, ya que el coeficiente de determinación $R^{2}$ fue de 0.96 . La fotólisis reveló un efecto poco significativo a la intensidad de $100 \mathrm{~mW} /$ $\mathrm{cm}^{2}$, por lo que se despreció frente a la fotocatálisis. La velocidad de degradación se incrementó conforme la dosis de $\mathrm{TiO}_{2}$ aumentó en el intervalo 0.8-1.0 g/L, pero disminuyó después desde 1.0 a $1.5 \mathrm{~g} / \mathrm{L}$ a un $\mathrm{pH}$ de $6 \mathrm{y}$ $100 \mathrm{~mW} / \mathrm{cm}^{2}$. La razón que se expuso fue la aglomeración de las partículas suspendidas y la protección de la luz. La remoción de $100 \%$ hallada se exhibió con la concentración inicial de ACE-K de 10 y 20 mg/L dentro de los 30 minutos. Contrariamente, el 95,89 y $82 \%$ fue conseguido con 30,40 y $50 \mathrm{mg} / \mathrm{L}$, respectivamente. La constante de velocidad de degradación $\left(k_{\mathrm{r}}\right)$ fue dependiente de la intensidad de la luz incidente, en concordancia con una ley de potencia fraccionaria de 0.8.

\section{Oxidación por Ultravioleta/Peróxido}

La fotólisis de $\mathrm{H}_{2} \mathrm{O}_{2}$ por acción de radiación UV, emitida por una lámpara de vapor de mercurio, en fase acuosa, se llevó a cabo desde 1910, como ha sido descrito por Stefan ${ }^{[46]}$. En 1929, Urey et al. ${ }^{[47]}$ determinaron que cualquiera de las siguientes reacciones es posible:

$$
\begin{gathered}
\mathrm{H}_{2} \mathrm{O}_{2}+h v \longrightarrow 2 \mathrm{HO}^{\circ} \\
\mathrm{H}_{2} \mathrm{O}_{2}+h v \longrightarrow \mathrm{H}_{2} \mathrm{O}+\frac{1}{2} \mathrm{O}_{2} \\
\mathrm{H}_{2} \mathrm{O}_{2}+h v \longrightarrow \mathrm{HO}_{2}^{\cdot}+\mathrm{H}^{\cdot}
\end{gathered}
$$

Los autores postularon que la absorción que experimenta el $\mathrm{H}_{2} \mathrm{O}_{2}$, en estado vapor, durante la iluminación, produce radicales $\cdot \mathrm{OH}$ conforme a la reacción (15). Más recientemente se ha reconocido la ruptura homolítica del enlace $\mathrm{O}-\mathrm{O}$, a longitudes de onda que oscilan entre 200 a $300 \mathrm{~nm}$, que conduce a una serie de reacciones sucesivas clasificadas en tres etapas ${ }^{[48,49]}$ :

Iniciación:

$$
\mathrm{H}_{2} \mathrm{O}_{2}+h v \longrightarrow 2 \mathrm{HO}
$$

Propagación:

$$
\begin{gathered}
\mathrm{HO}^{-}+\mathrm{H}_{2} \mathrm{O}_{2} \longrightarrow \mathrm{H}_{2} \mathrm{O}+\mathrm{HO}_{2}^{-} \\
\mathrm{HO}_{2}^{-}+\mathrm{H}_{2} \mathrm{O}_{2} \longrightarrow \mathrm{HO}^{-}+\mathrm{H}_{2} \mathrm{O}+\mathrm{O}_{2} \\
\mathrm{HO}^{-}+\mathrm{HO}_{2}^{-} \longrightarrow \mathrm{HO}_{2}^{-}+\mathrm{HO}^{-}
\end{gathered}
$$

Terminación:

$$
\begin{gathered}
2 \mathrm{HO}_{2}^{\cdot} \longrightarrow \mathrm{H}_{2} \mathrm{O}_{2}+\mathrm{O}_{2} \\
\mathrm{HO}^{\cdot}+\mathrm{HO}_{2}^{\cdot} \longrightarrow \mathrm{H}_{2} \mathrm{O}+\mathrm{O}_{2} \\
2 \mathrm{HO}^{\cdot} \longrightarrow \mathrm{H}_{2} \mathrm{O}_{2}
\end{gathered}
$$

La dosis óptima de $\mathrm{H}_{2} \mathrm{O}_{2}$ depende de factores como 1) calidad del agua, 2) tipo de lámpara e intensidad, 3) diseño del reactor, 4) reactividad del contaminante hacia - OH y 5) contribución por fotólisis directa ${ }^{[46]}$. Asimismo, la tasa de producción de radicales libres depende del pH y de las características de las lámparas UV y del medio, por ejemplo, la turbiedad.

Especial atención requiere este tratamiento en cuanto a la dosis debido a que el reactivo tiene una baja absortividad molar ${ }^{[24]}$, la cual indica cuánta luz es absorbida a 
una longitud de onda específica por una sustancia ${ }^{[50]}$. Por consiguiente, una concentración alta de $\mathrm{H}_{2} \mathrm{O}_{2}$ es demandada para una oxidación eficiente.

Lester et al. ${ }^{\left[{ }^{[1]}\right.}$ sometieron a oxidación avanzada a la SUC con el propósito de indicador conservativo dentro de una serie de compuestos orgánicos traza (COTr). Los dos tratamientos productores de radicales $\cdot \mathrm{OH}$ utilizados, $\mathrm{UV} / \mathrm{H}_{2} \mathrm{O}_{2}$ y $\mathrm{UV} / \mathrm{NO}_{3}$, se ejecutaron a escala piloto y laboratorio. En esta última, se dispuso de un reactor con una lámpara UV de baja presión montada en el centro, que emite a $253.7 \mathrm{~nm}$. Un régimen de recirculación se impuso a un flujo volumétrico de $6 \mathrm{~L} /$ min sobre la capacidad de $4 \mathrm{~L}$ del reactor. El agua tratada era potable o del efluente del tratamiento secundario de agua residual.

Los diez compuestos que se seleccionaron son lamotrigina, gemfibrozil, claritromicina, atenolol, ácido 2,4-diclorofenoxiacético, carbamazepina, metoprolol, trimetoprima, venlafaxina, difenhidramina, además de la SUC. Después de precalentar la lámpara durante 10 minutos, se agregaron los contaminantes para alcanzar $100 \mu \mathrm{g} / \mathrm{L}$ y $\mathrm{H}_{2} \mathrm{O}_{2}$ a una dosis de $10 \mathrm{mg} / \mathrm{L}$. Las muestras de $5 \mathrm{~mL}$ se extrajeron en diversos instantes. Referente a la escala piloto, localizada en la ciudad de Boulder, se incluyeron reactores conectados en paralelo con una lámpara UV de baja presión de $1000 \mathrm{~W}$ y otra de 400 $\mathrm{W}$ de media presión. El bombeo hacia los reactores del agua residual procedió desde un tanque ecualizador a razón de 1.9 o $3.8 \mathrm{~L} / \mathrm{min}$. La concentración de $\mathrm{H}_{2} \mathrm{O}_{2}$ administrada también fue de $10 \mathrm{mg} / \mathrm{L}$.

A escala laboratorio, la cinética de degradación siguió el modelo de pseudo primer orden con la velocidad de decaimiento más lenta en dos conformaciones: 1) SUC contra todos los COTr y 2) en el efluente de agua residual comparado con el agua de la llave. La relación de primer orden de los contaminantes objetivo y SUC, COTr/SUC, estuvo en el rango de 1.9 - 4.7 en agua de la llave y 1.3 - 4.3 en agua residual, con valores comunes $>2$ para la mayoría de los compuestos, excepto lamotrigina.

En la configuración del sistema piloto nuevamente la degradación de SUC fue la más deficiente cuando hubo una concentración de $19.26 \mu \mathrm{g} / \mathrm{L}$, excluyendo la claritromicina. En ambos escenarios, la predicción de la remoción de los $\mathrm{COTr}$ mediante $\mathrm{UV} / \mathrm{H}_{2} \mathrm{O}_{2}$ pudo sustentarse en la SUC como indicador conservativo.
Xu et al. ${ }^{[52]}$ examinaron la mineralización de SUC en fase acuosa sujeta a fotólisis en las modalidades UV, $\mathrm{UV} /$ peroxidisulfato (UV/PDS) y $\mathrm{UV} / \mathrm{H}_{2} \mathrm{O}_{2}$. Las variables básicas tomadas en cuenta fueron el $\mathrm{pH}$, la concentración de oxidante y SUC, así como los aniones inorgánicos $\mathrm{NO}_{2}^{-}, \mathrm{NO}_{3}^{-}$y $\mathrm{Cl}^{-}$para la eliminación de COT. Los ensayos se practicaron en un contenedor que alojaba en el centro una lámpara UV de $254 \mathrm{~nm}$ y $6 \mathrm{~W}$, orientada verticalmente e inmersa dentro de la solución. La temperatura de $25{ }^{\circ} \mathrm{C}$ fue controlada circulando agua de enfriamiento. La intensidad de radiación promedio se propuso a $7.07 \times 10^{-5}$ Einstein $/ \mathrm{m}^{2} \cdot \mathrm{s}$. El volumen de $250 \mathrm{~mL}$ de solución se agitó continuamente y el pH fue mantenido en 7 con una solución buffer de fosfato, omitiendo aquellas pruebas en la que se determinó el efecto del $\mathrm{pH}$.

La mineralización de SUC con UV/PDS y UV/ $\mathrm{H}_{2} \mathrm{O}_{2}$ siguió un patrón cinético de pseudo primer orden, validado por un coeficiente de correlación de $R^{2} \geq 0.95$, para un $\mathrm{pH}$ de $3,5,7,9$ y 11 . Por otra parte, la eficiencia particular de cada proceso exhibida fue diferente. En el caso de UV/PDS, la mejor mineralización de SUC se observó a condiciones ácidas acompañada por una remoción de COT de 75\% a pH 3 en 60 minutos, pero disminuyó a $64 \%$ con un $\mathrm{pH}$ de 7.

Una situación distinta sucedió con el sistema UV/ $\mathrm{H}_{2} \mathrm{O}_{2}$ : la eficiencia aumentó desde el pH de 3 a 7 y disminuyó en condiciones alcalinas. El mejor porcentaje de $64 \%$ se notó a un $\mathrm{pH} 7$, que se convirtió en $29 \%$ a un valor de $\mathrm{pH}$ de 11 . La presencia $<10 \mathrm{mM}$ de $\mathrm{Cl}^{-}$ en $\mathrm{UV} / \mathrm{H}_{2} \mathrm{O}_{2}$ promovió la mineralización, llevándola a una concentración mayor. $\mathrm{El} \mathrm{NO}_{3}^{-}$redujo la remoción, puesto que disminuyó de 64 a $40 \%$ conforme la concentración cambió de 0 a $200 \mathrm{mM}$. El $\mathrm{NO}_{2}^{-}$inhibió casi completamente la mineralización en ambos sistemas, provocando que la constante $k$ del modelo cinético llegara a $0.0005 \mathrm{~min}^{-1}$.

Con los dos oxidantes valorados, la mayor eficiencia en 60 minutos fue conseguida por UV/PDS, lo que removió el 93\% de COT, usando $2.52 \mathrm{mM}$ de oxidante a $\mathrm{pH} 7$, superior a $69 \%$ con $\mathrm{UV} / \mathrm{H}_{2} \mathrm{O}_{2}$. El incremento de la concentración de SUC de 0.05 a $0.252 \mathrm{mM}$ propició una disminución en la remoción de $47 \%$ con $\mathrm{UV} / \mathrm{H}_{2} \mathrm{O}_{2}$ después de 60 minutos y $3.78 \mathrm{mM}$ de oxidante. La propuesta UV/PDS fue más eficiente que $\mathrm{UV} / \mathrm{H}_{2} \mathrm{O}_{2}$ en el tratamiento del edulcorante artificial sucralosa. 


\section{E. Oxidación por Ultravioleta/Ozono}

El ozono $\left(\mathrm{O}_{3}\right)$ en solución acuosa absorbe radiaciones UV a partir de una longitud de onda de $200 \mathrm{~nm}$, llegando hasta $360 \mathrm{~nm}$, con el máximo de absorción en $253.7 \mathrm{~nm}{ }^{[49,53]}$. Se ha postulado que la fotólisis de las moléculas de $\mathrm{O}_{3}$ producen directamente $\mathrm{H}_{2} \mathrm{O}_{2}$. Posteriormente, el $\mathrm{H}_{2} \mathrm{O}_{2}$ puede experimentar una reacción de fotólisis para formar dos radicales $\cdot \mathrm{OH}$, como se muestra enseguida ${ }^{[54,55]}$ :

$$
\begin{gathered}
\mathrm{O}_{3}+\mathrm{H}_{2} \mathrm{O}+h v \longrightarrow \mathrm{H}_{2} \mathrm{O}_{2} \\
\mathrm{H}_{2} \mathrm{O}_{2}+h v \longrightarrow 2 \mathrm{HO}^{\circ}
\end{gathered}
$$

En una segunda ruta, el radical $\cdot \mathrm{OH}$ podría disociarse en $\mathrm{HO}_{2}^{*}$ por la acción del $\mathrm{O}_{3}$ para participar en otras reacciones en cadena ${ }^{[48,49]}$ :

$$
\begin{gathered}
\mathrm{O}_{3}+\mathrm{H}_{2} \mathrm{O}+h v \longrightarrow 2 \mathrm{HO}^{\bullet}+\mathrm{O}_{2} \\
\mathrm{O}_{3}+\mathrm{HO}^{\bullet} \longrightarrow 2 \mathrm{HO}_{2}^{\cdot}+\mathrm{O}_{2} \\
\mathrm{O}_{3}+\mathrm{HO}_{2}^{\cdot} \longrightarrow \mathrm{HO}^{\cdot}+2 \mathrm{O}_{2} \\
\mathrm{HO}^{\cdot}+\mathrm{HO}_{2}^{\cdot} \longrightarrow \mathrm{H}_{2} \mathrm{O}+\mathrm{O}_{2} \\
2 \mathrm{HO}^{\cdot} \longrightarrow \mathrm{H}_{2} \mathrm{O}_{2}
\end{gathered}
$$

Se ha enunciado que la mayor velocidad de destrucción orgánica se debe a la descomposición de ozono catalizada por $\mathrm{H}_{2} \mathrm{O}_{2}{ }^{[53]}$, según las reacciones anteriores.

$\mathrm{Xu}$ et al. ${ }^{[56]}$ investigaron la mineralización de SUC empleando $\mathrm{UV} / \mathrm{O}_{3}$ sujeto a estas cinco condiciones operativas: 1) $\mathrm{pH}, 2$ ) concentración de $\mathrm{O}_{3}, 3$ ) flujo volumétrico gaseoso $(Q), 4)$ concentración inicial de SUC y 5) aniones $\mathrm{Cl}^{-}$y $\mathrm{NO}_{3}^{-}$. Los experimentos se realizaron en un reactor de vidrio cilíndrico encamisado con una lámpara UV instalada verticalmente en el centro. La intensidad de radiación de la luz UV determinada en promedio equivalió a $33.4 \mathrm{~W} / \mathrm{m}^{2}$. La concentración de SUC de $50 \mathrm{mg} / \mathrm{L}$, equivalente a $18.11 \mathrm{mg} / \mathrm{L}$ de COT, se mantuvo en un $\mathrm{pH}$ de 7 usando solución buffer de fosfato de $50 \mathrm{mM}$. La velocidad de 450 revoluciones por minuto con el agitador magnético permitió homogeneizar las soluciones. El ozono que se creó con una descarga eléctrica sobre oxígeno seco, con pureza del 99\%, se burbujeó en el líquido al mismo tiempo que la luz UV fue encendida. El agua recirculada por medio de una bomba mantuvo la temperatura de reacción constante a $25^{\circ} \mathrm{C}$. La cinética se definió como de pseu- do orden cero, puesto que el $\mathrm{O}_{3}$ que ingresa continuamente al reactor ocasiona la permanente saturación del medio. Los resultados verificaron el supuesto con $R^{2}$, oscilando de 0.972 a 0.996 . La fotólisis directa con UV-C prácticamente no removió SUC en 60 minutos, confirmado una disminución irrelevante de COT.

En contraste, la mineralización obtenida con $\mathrm{UV} / \mathrm{O}_{3}$ llegó a $89.8 \%$ y únicamente con $\mathrm{O}_{3}$ a $39.1 \%$. El flujo gaseoso mostró una máxima remoción de COT de $92.1 \%$ sobre 120 minutos cuando se ajustó a $40 \mathrm{~L} / \mathrm{h}$. Asimismo, la mineralización mejoró, aumentando la concentración de gas $\mathrm{O}_{3}$ desde 19.4 a $25 \mathrm{mg} / \mathrm{L}$, corroborándose con las cinéticas 0.080 y $0.143 \mathrm{mg} / \mathrm{L} \cdot \mathrm{min}$, respectivamente, si la SUC, el flujo y $\mathrm{pH}$ son iguales a $50 \mathrm{mg} / \mathrm{L}, 35 \mathrm{~L} / \mathrm{h}$ y 7 . La velocidad de mineralización aumentó acorde a la variación de $\mathrm{pH}$ 3-11, según los valores $0.071-0.085 \mathrm{mg} / \mathrm{L} \cdot \mathrm{min}$.

Las concentraciones iniciales de SUC, 50, 75 y $100 \mathrm{mg} / \mathrm{L}$, situaron las cinéticas en $0.08,0.07$ y $0.051 \mathrm{mg} / \mathrm{L} \cdot \mathrm{min}$, lo que se explicó en consonancia con una relación molar más baja de $\cdot \mathrm{OH}$ a SUC. $1 \mathrm{mM}$ de $\mathrm{Cl}^{-1}$ operó la velocidad como $0.091 \mathrm{mg} / \mathrm{L} \cdot \mathrm{min}$ y $0.08 \mathrm{mg} / \mathrm{L} \cdot \mathrm{min}$ en ausencia de $\mathrm{Cl}^{-1}$, pero fue restringida con el incremento de 10 a $300 \mathrm{mM}$. Las cinéticas aumentaron desde 0.08 hasta $0.108 \mathrm{mg} / \mathrm{L} \cdot \mathrm{min}$ con las dosis en el rango de 0 a $200 \mathrm{mM}$, cuando se adicionó $\mathrm{NO}_{3}^{-}$. La oxidación de SUC se atribuyó principalmente a la reacción del edulcorante con los radicales $\cdot \mathrm{OH}$, los cuales se detectaron en los espectros de fluorescencia y cromatogramas de iones totales.

En la Tabla 1 se recopilan las mejores eficiencias de remoción de los edulcorantes, pertinentes a los POA del presente manuscrito, aunado a las condiciones experimentales especiales con las que se cumplen, según lo ejecutado por los investigadores.

En un contexto general, la electrooxidación es uno de los Procesos de Oxidación Avanzada en medio homogéneo que demanda energía ${ }^{[15]}$, con un sinnúmero de publicaciones hasta la fecha. Específicamente, se podría emplear el procedimiento reiteradamente para la degradación de edulcorantes artificiales porque la generación de radicales $\bullet \mathrm{OH}$ sucedería con una disposición básica de dos electrodos y el suministro de corriente eléctrica. 
TABLA 1.

Eficiencia de Remoción de Edulcorantes Artificiales del Agua por Diferentes POA

\begin{tabular}{|c|c|c|c|c|}
\hline MÉTOdo & EDULCORANTE & Mejor Escenario para Remoción & $\begin{array}{l}\text { EFICIENCIA DE } \\
\text { REMOCIÓN }\end{array}$ & REFERENCIA \\
\hline Electrooxidación & $\begin{array}{l}\text { Acesulfamo } \\
\text { de potasio } \\
(\mathrm{ACE}-\mathrm{K})\end{array}$ & $\begin{array}{l}100 \mathrm{mg} / \mathrm{L} \text { de ACE-K, } j=0.1 \mathrm{~A} / \mathrm{cm}^{2} \text {, electrolito }=1 \mathrm{M} \text { de } \\
\mathrm{Na}_{2} \mathrm{SO}_{4}, \mathrm{DDB}=2 \mathrm{~cm}^{2} \text { de área y } T=25^{\circ} \mathrm{C} . t=30 \text { minutos. }\end{array}$ & $100 \%$ & 21 \\
\hline \multirow[t]{4}{*}{ Electro-Fenton } & $\begin{array}{l}\text { Aspartamo } \\
\text { (ASP) }\end{array}$ & $\begin{array}{l}0.2 \mathrm{mM} \text { de } \mathrm{ASP}, \mathrm{I}=200 \mathrm{~mA},\left[\mathrm{Fe}^{2+}\right]=0.2 \mathrm{mM}, \mathrm{pH}_{0}=3 \\
{\left[\mathrm{Na}_{2} \mathrm{SO}_{4}\right]=50 \mathrm{mM} \text {, ánodo: } \mathrm{DDB} \text {, cátodo: fieltro de carbono. }} \\
t=15 \text { minutos. }\end{array}$ & $100 \%$ & 30 \\
\hline & $\begin{array}{l}\text { Sucralosa } \\
\text { (SUC) }\end{array}$ & $\begin{array}{l}0.2 \mathrm{mM} \text { de SUC, } \mathrm{I}=200 \mathrm{~mA},\left[\mathrm{Fe}^{2+}\right]=0.2 \mathrm{mM}, \mathrm{pH}_{0}=3 \\
{\left[\mathrm{Na}_{2} \mathrm{SO}_{4}\right]=50 \mathrm{mM} \text {, ánodo: } \mathrm{DDB} \text {, cátodo: fieltro de carbono. }} \\
t=120 \text { minutos. }\end{array}$ & $96.1 \%$ de COT & 31 \\
\hline & Sacarina (SAC) & $\begin{array}{l}0.2 \mathrm{mM} \text { de } \mathrm{SAC}, \mathrm{I}=200 \mathrm{~mA},\left[\mathrm{Fe}^{2+}\right]=0.2 \mathrm{mM}, \mathrm{pH}_{0}=3, \\
{\left[\mathrm{Na}_{2} \mathrm{SO}_{4}\right]=50 \mathrm{mM} \text {, ánodo: } \mathrm{DDB} \text {, cátodo: fieltro de carbono. }} \\
t=120 \text { minutos. }\end{array}$ & $96.2 \%$ de COT & 32 \\
\hline & SAC & $\begin{array}{l}0.2 \mathrm{mM} \text { de } \mathrm{SAC}, \mathrm{I}=200 \mathrm{~mA},\left[\mathrm{Fe}^{2+}\right]=0.2 \mathrm{mM}, \mathrm{pH}_{0}=3, \\
{\left[\mathrm{Na}_{2} \mathrm{SO}_{4}\right]=50 \mathrm{mM} \text { o }\left[\mathrm{NaNO}_{3}\right]=100 \mathrm{mM} \text {, ánodo: } \mathrm{DDB}, \mathrm{Pt} \mathrm{o}} \\
\text { ADS, cátodo: fieltro de carbono. } t=25 \text { minutos. }\end{array}$ & Casi $100 \%$ & 34 \\
\hline Foto-Fenton & ACE & $\begin{array}{l}75 \mu \mathrm{m} \text { de } \mathrm{ACE}, \mathrm{pH}=3, \mathrm{H}_{2} \mathrm{O}_{2}=750 \mathrm{Mm},\left[\mathrm{Fe}^{2+}\right]_{0}=150 \mu \mathrm{M} \text {, } \\
\text { irradiación }=2.2 \mathrm{~mW} / \mathrm{cm}^{2} \text { UVA. } t=5 \text { minutos. }\end{array}$ & $100 \%$ & 38 \\
\hline \multirow[t]{2}{*}{$\begin{array}{l}\text { Fotocatálisis } \\
\text { heterogénea }\end{array}$} & ACE-K & $\begin{array}{l}20 \mathrm{mg} / \mathrm{L} \text { de } \mathrm{ACE}-\mathrm{K}, 100 \mathrm{mg} / \mathrm{L} \text { de catalizador: } \mathrm{ZnO} \text { dopado } \\
\text { con } 1 \% \text { de } \mathrm{Ce}\left(\mathrm{SO}_{4}\right)_{2} \text {, intensidad de radiación }=750 \mathrm{~W} / \mathrm{m}^{2} \text {, } \\
\mathrm{T}<20^{\circ} \mathrm{C} . t=10 \text { minutos irradiación. }\end{array}$ & $\begin{array}{l}68 \% \text { agua ultrapura } \\
42 \% \text { agua de río }\end{array}$ & 43 \\
\hline & ACE-K & $\begin{array}{l}10 \text { y } 20 \mathrm{mg} / \mathrm{L} \text { de } \mathrm{ACE}-\mathrm{K}, \mathrm{pH}=6,[\mathrm{TiO} 2]=1 \mathrm{~g} / \mathrm{L}, \text { intensidad } \\
\text { de irradiación }=100 \mathrm{~mW} / \mathrm{cm} 2 \text {, adsorción previa }=1 \text { hora. } \\
t=30 \text { minutos. }\end{array}$ & $100 \%$ & 45 \\
\hline \multirow[t]{2}{*}{$\mathrm{UV} / \mathrm{H}_{2} \mathrm{O}_{2}$} & SUC & $\begin{array}{l}\text { [Contaminantes] }=100 \mu \mathrm{g} / \mathrm{L} \text {, fluencias UV: } 750 \text { y } 1500 \mathrm{~mJ} / \\
\mathrm{cm}^{2} \text {, efluente tratamiento secundario: } 3.8 \text { y } 1.9 \mathrm{~L} / \mathrm{min}, \mathrm{H}_{2} \mathrm{O}_{2} \text { : } \\
\text { dosis } 10 \mathrm{mg} / \mathrm{L} \text {. }\end{array}$ & $\begin{array}{l}\text { Lampara UV: } \\
\text { Media presión: } 61 \% \text {, } \\
750 \mathrm{~mJ} / \mathrm{cm}^{2} \text { y } 65 \% \text {, } \\
1500 \mathrm{~mJ} / \mathrm{cm}^{2} \\
\text { Baja presión: } 73 \% \text {, } \\
750 \mathrm{~mJ} / \mathrm{cm}^{2} \text { y } 92 \% \text {, } \\
1500 \mathrm{~mJ} / \mathrm{cm}^{2}\end{array}$ & 51 \\
\hline & SUC & $\begin{array}{l}0.126 \mathrm{mM} \text { de } \mathrm{SUC},\left[\mathrm{H}_{2} \mathrm{O}_{2}\right]=3.78 \mathrm{mM}, \mathrm{pH}=7, \text { intensidad de } \\
\text { radiación }=7.07 \times 10^{-5} \text { Einstein } / \mathrm{m}^{2} \cdot \mathrm{s} . t=60 \text { minutos }\end{array}$ & $75 \%$ como COT & 52 \\
\hline $\mathrm{UV} / \mathrm{O}_{3}$ & SUC & $\begin{array}{l}50 \mathrm{mg} / \mathrm{L} \text { de SUC, }\left[\mathrm{O}_{3}\right]=19.4 \mathrm{mg} / \mathrm{L} \text {, flujo } \mathrm{O}_{3}=35 \mathrm{~L} / \text { hora, } \mathrm{pH} \\
=11 \text {, intensidad de radiación }=33.4 \mathrm{~W} / \mathrm{m}^{2} . t=120 \text { minutos. }\end{array}$ & $93 \%$ como COT & 56 \\
\hline
\end{tabular}

En un contexto general, la electrooxidación es uno de los Procesos de Oxidación Avanzada en medio homogéneo que demanda energía ${ }^{[15]}$, con un sinnúmero de publicaciones hasta la fecha. Específicamente, se podría emplear el procedimiento reiteradamente para la degradación de edulcorantes artificiales porque la generación de radicales $\cdot \mathrm{OH}$ sucedería con una disposición básica de dos electrodos y el suministro de corriente eléctrica.

\section{CONCLUSIONES}

El destino final de todos los edulcorantes artificiales, después que se han consumido, es el agua. A pesar de que todos los POA evaluados son capaces de generar efectivamente radicales $\cdot \mathrm{OH}$ para lograr una degradación importante, los autores de las publicaciones se concentraron solamente en acesulfamo, aspartamo, sacarina y, en la mayoría de ellas, sucralosa.

A partir de este hecho se argumenta que más investigación se puede llevar a cabo en tres direcciones: 1) la remoción de otros edulcorantes que ya han sido identificados en matrices acuosas o que han sido aprobados para su utilización: neohesperidina dihidrocalcona (NHDC), ciclamato, neotamo, alitamo, D-psicosa, D-tagatosa, entre otros; 2) la evaluación de otros $\mathrm{POA}$, como energía ultrasónica acoplada con oxidantes $\left(\mathrm{H}_{2} \mathrm{O}_{2}, \mathrm{O}_{3}\right)$ 
o procesos heterogéneos con $\mathrm{O}_{3}$ y 3 ) las variables principales involucradas para la ejecución también se pueden examinar con el propósito de definir condiciones óptimas. Las cantidades encontradas servirían como base para la implementación a escala piloto o real según lo permita la tecnología que prevalezca.

Si bien no se efectuó en cada uno de los estudios o no se especificó con detalle en esta revisión, la elucidación de los productos de transformación, también conocidos como subproductos, fue meritoria para conocer el alcance que tienen los POA. Por lo tanto, continuar con esa práctica en futuras investigaciones será crucial para predecir los compuestos que podrían surgir en medios acuáticos naturales como consecuencia de fenómenos oxidativos incompletos.

\section{REFERENCIAS}

[1] M. Carocho, P. Morales y I. C. F. R. Ferreira, "Sweeteners as food additives in the XXI century: A review of what is known, and what is to come", Food and Chemical Toxicology, vol. 107A, pp. 302-317, Septiembre 2017. https://doi.org/10.1016/j.fct.2017.06.046

[2] S. Marie, "Sweeteners", en Food Additive User's Handbook, 1. a ed. J. Smith, ed. Nueva York: Springer, 1995, pp. 47-74. https://doi.org/10.1007/978-1-4757-5247-2

[3] A. Das y R. Chakraborty, "An Introduction to Sweeteners", en Sweeteners: Pharmacology, Biotechnology, and Applications, 1. a ed. J. M. Mérillon y K. G. Ramawat, eds. Cham, Suiza: Springer, 2018, pp. 1-13. https: //doi. org/10.1007/978-3-319-27027-2

[4] S. Chattopadhyay, U. Raychaudhuri y R. Chakraborty, "Artificial sweeteners - a review", Journal of Food Science and Technology, vol. 51, no. 4, pp. 611-621, Abril 2014. https://doi.org/10.1007/s13197-011-0571-1

[5] R. H. Mazur, A. H. Goldkamp, P. A. James y J. M. Schlatter, "Structure-taste relation of aspartic acid amides", Journal of Medicinal Chemistry, vol. 13, no. 6, pp. 1217-1221, Noviembre 1970. https://doi.org/10.1021/ jm00300a046

[6] D. Benton, "Can artificial sweeteners help control body weight and prevent obesity?", Nutrition Research Reviews, vol. 18, no. 1, pp. 63-76, Junio 2005. https://doi. org/10.1079/NRR200494
[7] M. Salimi, A. Esrafili, M. Gholami, A. J. Jafari, R. R. Kalantary, M. Farzadkia, M. Kermani y H. R. Sobhi, "Contaminants of emerging concern: a review of new approach in AOP technologies", Environmental Monitoring and Assessment, vol. 189, no. 414, pp. 1-22, Agosto 2017. https://doi.org/10.1007/s10661-017-6097-x

[8] M. G. Lindley, "Sweetener markets, marketing and product development", en Handbook of Sweeteners, 1. ${ }^{a}$ ed. S. Marie y J. R. Piggot, eds. Boston: Springer, 1991, pp. 186204. https://doi.org/10.1007/978-1-4757-5380-6

[9] N. H. Tran, J. Hu, J. Li y S. L. Ong, "Suitability of artificial sweeteners as indicators of raw wastewater contamination in surface water and groundwater", Water Research, vol. 48, pp. 443-456, Enero 2014. https://doi. org/10.1016/j.watres.2013.09.053

[10] Z. Sang, Y. Jiang, Y-K Tsoi y K. S-Y. Leung, "Evaluating the environmental impact of artificial sweeteners: A study of their distributions, photodegradation and toxicities", Water Research, vol. 52, pp. 260-274, Abril 2014. https://doi.org/10.1016/j.watres.2013.11.002

[11] F. T. Lange, M. Scheurer y H-J. Brauch, "Artificial sweeteners-a recently recognized class of emerging environmental contaminants: a review", Analytical and Bioanalytical Chemistry, vol. 403, no. 9, pp. 2503-2518, Julio 2012. https://doi.org/10.1007/s00216-012-5892-z

[12] J. Luo, Q. Zhang, M. Cao, L. Wud, J. Cao, F. Fang, C. Li, Z. Xue y Q. Feng, "Ecotoxicity and environmental fates of newly recognized contaminants artificial sweeteners: A review", Science of The Total Environment, vol. 653, pp. 1149-1160, Febrero 2019. https://doi.org/10.1016/j. scitotenv.2018.10.445

[13] Y. Deng y R. Zhao, "Advanced Oxidation Processes (AOPs) in Wastewater Treatment", Current Pollution Reports, vol. 1, no. 3, pp. 167-176, Septiembre 2015. https://doi.org/10.1007/s40726-015-0015-Z

[14] W. H. Glaze, J-W. Kang y D. H. Chapin, "The Chemistry of Water Treatment Processes Involving Ozone, Hydrogen Peroxide and Ultraviolet Radiation", Ozone: Science \& Engineering, vol. 9, no. 4, pp. 335-352, 1987. https:// doi.org/10.1080/01919518708552148

[15] A. A. Mayyahi y H. A. A. Al-Asadi, "Advanced Oxidation Processes (AOPs) for Wastewater Treatment and 
Reuse: A Brief Review", Asian Journal of Applied Science and Technology, vol. 2, no. 3, pp. 18-30, Julio-Septiembre 2018. http://ajast.net/data/uploads/6002.pdf

[16] C. Barrera-Díaz, P. Cañizares, F. J. Fernández, R. Natividad, y M. A. Rodrigo, "Electrochemical Advanced Oxidation Processes: An Overview of the Current Applications to Actual Industrial Effluents", Journal of the Mexican Chemical Society, vol. 58, no. 3, pp. 256-275, 2014. https://doi.org/10.29356/jmcs.v58i3.133

[17] S. Garcia-Segura, J. D. Ocon y M. N. Chong, "Electrochemical oxidation remediation of real wastewater effluents - A review", Process Safety and Environmental Protection, vol. 113, pp. 48-67, Enero 2018. https://doi. org/10.1016/j.psep.2017.09.014

[18] C. A. Martínez-Huitle y M. Panizza, "Electrochemical oxidation of organic pollutants for wastewater treatment", Current Opinion in Electrochemistry, vol. 11, pp. 62-71, Octubre 2018. https://doi.org/10.1016/j. coelec.2018.07.010

[19] H. Särkkä, A. Bhatnagar y M Sillanpää, "Recent developments of electro-oxidation in water treatment - A review", Journal of Electroanalytical Chemistry, vol. 754, pp. 46-56, Octubre 2015. https://doi.org/10.1016/j. jelechem.2015.06.016

[20] C. A. Martínez-Huitle y S. Ferro, "Electrochemical oxidation of organic pollutants for the wastewater treatment: direct and indirect processes", Chemical Society Reviews, vol. 35, no. 12, pp. 1324-1340, 2006. https:// doi.org/10.1039/B517632H

[21] V. Punturat y K-L. Huang, "Degradation of acesulfame in aqueous solutions by electro-oxidation", Journal of the Taiwan Institute of Chemical Engineers, vol. 63, pp. 286-294, Junio 2016. https://doi.org/10.1016/j.jtice. 2016.03 .016

[22] C. Walling, "Fenton's Reagent Revisited", Accounts of Chemical Research, vol. 8, no. 4, pp. 125-131, Abril 1975. https://doi.org/10.1021/ar50088a003

[23] J. J. Pignatello, D. Liu y P. Huston, "Evidence for an Additional Oxidant in the Photoassisted Fenton Reaction", Environmental Science \& Technology, vol. 33, no. 11, pp. 1832-1839, Abril - Junio 1999. https://doi.org/10.1021/ es $980969 b$
[24] Black \& Veatch Corporation, "Advanced Oxidation Processes", en White's Handbook of Chlorination and Alternative Disinfectants, 5. ${ }^{\text {a }}$ ed. Black \& Veatch Corporation, ed. John Wiley and Sons, 2010, pp. 976-1002. https://doi.org/10.1002/9780470561331.ch18

[25] P. R. Gogate y A. B. Pandit, "A review of imperative technologies for wastewater treatment I: oxidation technologies at ambient conditions", Advances in Environmental Research, vol. 8, no. 3-4, pp. 501-551, Marzo 2004. https://doi.org/10.1016/S1093-0191(03)00032-7

[26] M. Panizza y G. Cerisola, "Removal of organic pollutants from industrial wastewater by electrogenerated Fenton's reagent", Water Research, vol. 35, no. 16, pp. 3987-3992, Noviembre 2001. https://doi.org/10.1016/ S0043-1354(01)00135-X

[27] S. Qiu, D. He, J. Ma, T. Liu y T. D. Waite, "Kinetic Modeling of the Electro-Fenton Process: Quantification of Reactive Oxygen Species Generation", Electrochimica Acta, vol. 176, pp. 51-58, Septiembre 2015. http://dx. doi.org/10.1016/j.electacta.2015.06.103

[28] A. Özcana, M. A. Oturan, N. Oturan y Y. Sahin, "Removal of Acid Orange 7 from water by electrochemically generated Fenton's reagent", Journal of Hazardous Materials, vol. 163, no. 2-3, pp. 1213-1220, Abril 2009. https://doi.org/10.1016/j.jhazmat.2008.07.088

[29] H. He y Z. Zhou, "Electro-Fenton process for water and wastewater treatment", Critical Reviews in Environmental Science and Technology, vol. 47, no. 21, pp. 21002131, Diciembre 2017. https://doi.org/10.1080/106433 89.2017.1405673

[30] H. Lin, N. Oturan, J. Wu, V. K. Sharma, H. Zhang y M. A. Oturan, "Removal of artificial sweetener aspartame from aqueous media by electrochemical advanced oxidation processes" Chemosphere, vol. 167, pp. 220-227, Enero 2017. https://doi.org/10.1016/j.chemosphere.20 16.09 .143

[31] H. Lin, N. Oturan, J. Wu, H. Zhang y M. A. Oturan, "Cold incineration of sucralose in aqueous solution by electro-Fenton process", Separation and Purification Technology, vol. 173, pp. 218-225, Febrero 2017. https:// doi.org/10.1016/j.seppur.2016.09.028 
[32] H. Lin, N. Oturan, J. Wu, M. A. Oturan y H. Zhang, “The application of electro-Fenton process for the treatment of artificial sweeteners", en Electro-Fenton Process. New Trends and Scale-Up, M. Zhou, M. A. Oturan y I. Sirés, eds. Singapur: Springer, 2018, pp. 379-398. https://doi. org/10.1007/978-981-10-6406-7

[33] S. Trasatti, "Electrocatalysis: understanding the success of DSA ${ }^{\infty}$, Electrochimica Acta, vol. 45, no. 15-16, pp. 2377-2385, Mayo 2000. https://doi.org/10.1016/S00134686(00)00338-8

[34] H. Lin, J. Wu, N. Oturan, H. Zhang y M. A. Oturan, "Degradation of artificial sweetener saccharin in aqueous medium by electrochemically generated hydroxyl radicals", Environmental Science and Pollution Research, vol. 23, no. 5, pp. 4442-4453, Marzo 2016. https://doi.org/10.1007/s11356-015-5633-x

[35] E. Brillas, I. Sirés y M. A. Oturan, "Electro-Fenton Process and Related Electrochemical Technologies Based on Fenton's Reaction Chemistry", Chemical Reviews, vol. 109, no. 12, pp. 6570-6631, Octubre 2009. https:// doi.org/10.1021/cr900136g

[36] S. R. Pouran, A. R. A. Aziz y W. M. A. W. Daud, "Review on the main advances in photo-Fenton oxidation system for recalcitrant wastewaters", Journal of Industrial and Engineering Chemistry, vol. 21, pp. 53-69, Enero 2015. https://doi.org/10.1016/j.jiec.2014.05.005

[37] S-M. Kim y A. Vogelpohl, "Degradation of Organic Pollutants by the Photo-Fenton-Process", Chemical Engineering Technology, vol. 21, no. 2, pp. 187-191, Febrero 1998. https://doi.org/10.1002/(SICI)1521-4125(199802) 21:2\%3C187::AID-CEAT187\%3E3.0.CO;2-H

[38] E. Kattel, M. Trapido y N. Dulova, “Oxidative degradation of emerging micropollutant acesulfame in aqueous matrices by UVA-induced $\mathrm{H}_{2} \mathrm{O}_{2} / \mathrm{Fe}^{2+}$ y $\mathrm{S}_{2} \mathrm{O}_{8}^{2-} / \mathrm{Fe}^{2+}$ processes", Chemosphere, vol. 171, pp. 528-536, Marzo 2017. https://doi.org/10.1016/j.chemosphere.2016.12.104

[39] P. A. Desario y K. A. Gray, "Passive systems to improve air quality and reduce heat retention in the urban environment", en Metropolitan Sustainability, Understanding and Improving the Urban Environment, Woodhead Publishing Series in Energy, 1. ${ }^{a}$ ed. F. Zeman ed. Woodhead Publishing, 2012, pp. 292-316. https://doi. org/10.1533/9780857096463.3.292
[40] R. A. Al-Rasheed, "Water treatment by heterogeneous photocatalysis an overview", en Proceedings of the 4th SWCC Acquired Experience Symposium [En línea], Jeddah, Saudi Arabia, 2005. Disponible en: http://citeseerx.ist.psu.edu/viewdoc/download?$\mathrm{doi}=10 \cdot 1 \cdot 1 \cdot 552.942 \& \mathrm{rep}=\mathrm{rep} 1 \&$ type $=$ pdf

[41] C. C. Kaan, A. A. Aziz, S. Ibrahim, M. Matheswaran y P. Saravanan, "Heterogeneous Photocatalytic Oxidation an Effective Tool for Wastewater Treatment - A Review”, en Studies on Water Management Issues, M. Kumarasamy, ed. Londres: IntechOpen, 2012, pp. 219-236. https://doi. org/10.5772/30134

[42] A. L. Linsebigler, G. Lu y J. T. Yates, "Photocatalysis on $\mathrm{TiO}_{2}$ surfaces: principles, mechanisms, and selected results", Chemical Reviews, vol. 95, no. 3, pp. 735-758, Mayo 1995. https://doi.org/10.1021/cr00035a013

[43] P. Calza, C. Gionco, M. Giletta, M. Kalaboka, V.A. Sakkas, T. Albanis y M.C. Paganini, "Assessment of the abatement of acelsulfame $\mathrm{K}$ using cerium doped $\mathrm{ZnO}$ as photocatalyst", Journal of Hazardous Materials, vol. 323, parte A, pp. 471-477, Febrero 2017. https://doi. org/10.1016/j.jhazmat.2016.03.093

[44] T. Ohno, K. Sarukawa, K. Tokieda y M. Matsumura, "Morphology of a $\mathrm{TiO}_{2}$ Photocatalyst (Degussa, P-25) Consisting of Anatase and Rutile Crystalline Phases", Journal of Catalysis, vol. 203, no. 1, pp. 82-86, Octubre 2001. https://doi.org/10.1006/jcat.2001.3316

[45] M. Ghosh, P. Chowdhury, A. K. Ray, "Study of solar photocatalytic degradation of Acesulfame $\mathrm{K}$ to limit the outpouring of artificial sweeteners", Separation and Purification Technology, vol. 207, pp. 51-57, Diciembre 2018. https://doi.org/10.1016/j.seppur.2018.05.062

[46] M. I. Stefan, "UV/Hydrogen peroxide process" en Advanced Oxidation Processes for Water Treatment. Fundamentals and Applications, M. I. Stefan, ed. Londres: IWA Publishing, 2017, pp. 7-122. https://doi. org/10.2166/9781780407197_0007

[47] H. C. Urey, L. H. Dawsey y F. O. Rice, "The absorption spectrum and decomposition of hydrogen peroxide by light", Journal of the American Chemical Society, vol. 51, no. 5, pp. 1371-1383, Mayo 1929. https://doi. org/10.1021/ja01380a011 
[48] F. Zaviska, P. Drogui, G. Mercier y J-F. Blais. "Procédés d'oxydation avancée dans le traitement des eaux et des effluents industriels: Application à la dégradation des polluants réfractaires", Revue des sciences de l'eau, vol. 22, no. 4, pp. 535-564, Octubre 2009. https://doi. org/10.7202/038330ar

[49] M. A. Oturan y J-J. Aaron, "Advanced Oxidation Processes in Water/Wastewater Treatment: Principles and Applications. A Review", Critical Reviews in Environmental Science and Technology, vol. 44, no. 23, pp. 2577-2641, Septiembre 2014. https://doi.org/10.1080/1 0643389.2013 .829765

[50] D. C. Harris y C. A. Lucy, "Fundamentals of Spectrophotometry", en Quantitative chemical analysis, 9. ${ }^{\mathrm{a}} \mathrm{ed}$. D. C. Harris, ed. Nueva York: W. H. Freeman \& Company, 2016, pp. 432-460.

[51] Y. Lester, I. Ferrer, E. M. Thurman y K. G. Linden, "Demonstrating sucralose as a monitor of full-scale UV/ AOP treatment of trace organic compounds", Journal of Hazardous Materials, vol. 280, pp. 104-110, Septiembre 2014. https://doi.org/10.1016/j.jhazmat.2014.07.009

[52] Y. Xu, Z. Lin y H. Zhang, "Mineralization of sucralose by UV-based advanced oxidation processes: UV/ PDS versus $\mathrm{UV} / \mathrm{H}_{2} \mathrm{O}_{2}$ ", Chemical Engineering Journal, vol. 285, pp. 392-401, Febrero 2016. https://doi.org/ 10.1016/j.cej.2015.09.091

[53] H. Zhou y D. W. Smith, "Advanced technologies in water and wastewater treatment", Journal of Environmental Engineering and Science, vol. 1, no. 4, pp. 247-264, Julio 2002. https://doi.org/10.1139/s02-020
[54] K. Ikehata y Y. Li, “Ozone-Based Processes", en Advanced Oxidation Processes for Wastewater Treatment, 1a. ed. S. C. Ameta y R. Ameta, eds. Academic Press, 2018, pp. 115-134. https://doi.org/10.1016/B978-0-12810499-6.00005-X

[55] F. J. Beltran, "Kinetics of the Ozone-UV Radiation System", en Ozone Reaction Kinetics for Water and Wastewater Systems, 1. ${ }^{\mathrm{a}}$ ed. F. J. Beltran, ed. Boca Raton: CRC Press, 2003, pp. 193-226. https://doi. org/10.1201/9780203509173

[56] Y. Xu, Y. Wu, W. Zhang, X. Fan, Y. Wang y H. Zhang, "Performance of artificial sweetener sucralose mineralization via $\mathrm{UV} / \mathrm{O}_{3}$ process: Kinetics, toxicity and intermediates", Chemical Engineering Journal, vol. 353, pp. 626-634, Diciembre 2018. https://doi.org/10.1016/j. cej.2018.07.090

[57] I. J. Buerge, M. Keller, H-R Buser, M. D. Müller y T. Poiger, "Saccharin and other artificial sweeteners in soils: estimated inputs from agriculture and households, degradation, and leaching to groundwater", Environmental Science \& Technology, vol. 45, no. 2, pp. 615-621, Enero 2011. https://doi.org/10.1021/es1031272

[58] S. M. Praveena, M. S. Cheema y H-R. Guo, "Non-nutritive artificial sweeteners as an emerging contaminant in environment: A global review and risks perspectives", Ecotoxicology and Environmental Safety, vol. 170, pp. 699-707, Abril 2019. https://doi.org/10.1016/j. ecoenv.2018.12.048 